\title{
Stability of $m$-equivalence to the weak Pinsker property
}

\author{
ADAM FIELDSTEEL \\ Wesleyan University, Middletown, CT 06457, USA \\ AND \\ DANIEL J. RUDOLPH \\ University of Maryland, College Park, MD 20742, USA
}

(Received 21 March 1988 and revised 29 June 1988)

\begin{abstract}
Let $\mathscr{W} \mathscr{P}$ denote the class of transformations with the weak Pinsker property, and let $[\mathscr{W} \mathscr{P}]_{m}$ denote the class of transformations $m$-equivalent to some member of $\mathscr{W P}$, where $m$ is an entropy-preserving size. We show that if $T$ is a factor of an element of $[\mathscr{W} \mathscr{P}]_{m}$, then $T \in[\mathscr{W P P}]_{m}$, and if $T$ is an $\bar{m}$-limit of elements of $[\mathscr{W} \mathscr{P}]_{m}$, then $T \in[\mathscr{W P}]_{m}$.
\end{abstract}

In this paper we apply the theory of restricted orbit equivalences of ergodic transformations, as developed in the recent memoir of Rudolph [2], to the study of the weak Pinsker property. We begin with a brief summary of some of the basic ideas in Rudolph's work.

Let $T$ be an ergodic transformation. (Throughout this paper, all transformations are assumed to be, or may be shown to be ergodic measure-preserving transformations of a Lebesgue probability space.) We define an integer-valued function $\alpha$ on the orbit relation determined by $T$ by $\alpha\left(\omega, \omega^{\prime}\right)=n$ if $T^{n}(\omega)=\omega^{\prime}$, and refer to $\alpha$ as an ordering. We may indicate the relation between $T$ and $\alpha$ by writing $T$ as $T_{\alpha}$. By an orbit equivalence we mean a pair of transformations $T$ and $T^{\prime}$ on the same space with the same orbits, hence giving (and being given by) two orderings. If $\alpha$ and $\alpha^{\prime}$ are two such orderings, the orbit equivalence they represent, denoted $\left(\alpha, \alpha^{\prime}\right)$, is called a coboundary if there is a measurable function $g$ such that off a set of orbits of measure zero, $\alpha\left(\omega, \omega^{\prime}\right)=\alpha^{\prime}\left(\omega, \omega^{\prime}\right)+g(\omega)-g\left(\omega^{\prime}\right)$. In this case, the map $\varphi: \omega \mapsto\left(T_{\alpha^{\prime}}\right)^{g(\omega)}(\omega)$ is an isomorphism between $T_{\alpha}$ and $T_{\alpha^{\prime}}$, so that from a dynamical point of view, a coboundary is a trivial orbit equivalence.

Each orbit equivalence ( $\left.\alpha, \alpha^{\prime}\right)$ may be viewed as providing, for each $\omega$, a permutation of the integers corresponding to the change from $\alpha$ to $\alpha^{\prime}$. This permutation can be described by the function $n \mapsto f_{\omega}^{\alpha, \alpha^{\prime}}(n)=\alpha^{\prime}\left(\omega, T_{\alpha}^{n}(\omega)\right)$, or in more local terms, by the permutations $\prod_{\omega,[a, b]}^{\alpha, \alpha^{\prime}}:[a, b] \rightarrow[a, b]$ which reorder intervals $[a, b]$ in $\mathbb{Z}$ in the same way that $\alpha^{\prime}$ reorders the points in the orbit segment $\left\{T_{\alpha}^{a} \omega, T_{\alpha}^{a+1} \omega, \ldots, T_{\alpha}^{b} \omega\right\}$. If $m$ is a non-negative, real-valued function defined on permutations of finite intervals 
in $\mathbb{Z}$, then by setting

$$
m\left(\alpha, \alpha^{\prime}\right)=\lim _{\substack{a \rightarrow-\infty \\ b \rightarrow \infty}} m\left(\prod_{\omega,[a, b]}^{\alpha, \alpha^{\prime}}\right)
$$

one extends $m$ to be defined on orbit equivalences. (Note $m$ is constant by ergodicity.) The function $m$ is called a size if it satisfies six mild conditions, Axioms (i) through (vi) of [2], which we will not reproduce here. Given a size $m$, an orbit equivalence $\left(\alpha, \alpha^{\prime}\right)$ is called an $m$-equivalence if it is a limit of coboundaries in the following sense: for every $\varepsilon>0$, there is a coboundary $\left(\alpha, \alpha^{\varepsilon}\right)$ such that $m\left(\alpha^{\prime}, \alpha^{\varepsilon}\right)<\varepsilon$. Transformations $T$ and $T^{\prime}$ are called $m$-equivalent if there is an $m$-equivalence ( $\alpha, \alpha^{\prime}$ ) such that $T_{\alpha}$ is isomorphic to $T$, and $T_{\alpha^{\prime}}$ is isomorphic to $T^{\prime}$. We should remark that each of the relations of isomorphism, even Kakutani equivalence, and (unrestricted) orbit equivalence is the relation of $m$-equivalence for an appropriate choice of $\boldsymbol{m}$.

A transformation $T$ is said to have the weak Pinsker property if for every $\varepsilon>0$, $T$ is isomorphic to a direct product $T_{1} \times T_{2}$ where $T_{1}$ is isomorphic to an independent process and $h\left(T_{2}\right)<\varepsilon$. No transformations are known to fail to have this property. J.-P. Thouvenot showed in [4] that this property is preserved under the taking of factors and $\bar{d}$-limits. We will establish analogous results for the property of $m$ equivalence to a transformation with the weak Pinsker property.

In [2] it is shown that sizes are subject to the following dichotomy. Either, for all transformations $T$, there exists a transformation $T^{\prime}$ such that $T^{\prime}$ is $m$-equivalent to $T$, and $h\left(T^{\prime}\right)=0$, or, for all $T$, if $T^{\prime}$ is $m$-equivalent to $T$, then $h\left(T^{\prime}\right)=h(T)$. Our concerns are only non-trivial in the latter case, so henceforth we assume that all sizes under discussion have this entropy-preserving character.

We will use several basic results concerning sizes. First, given a size $m$ and $\hat{\varepsilon}>0$ and an integer $K>0$, there is a $\delta>0$ (depending only on $m, \hat{\varepsilon}$, and $K$ ) such that if $\left(\alpha, \alpha^{\prime}\right)$ is an orbit equivalence with $m\left(\alpha, \alpha^{\prime}\right)<\delta$, then for a set of $\omega$ of measure greater that $1-\hat{\varepsilon}$, and all $k \in(-K, K), T_{\alpha}^{k}(\omega)=T_{\alpha^{\prime}}^{k}(\omega)$. This follows easily from Axiom (iv) and Lemma 2.2 of [2].

Next, suppose $\alpha$ is an ordering and $P$ is a finite partition giving rise to a process $\left(T_{\alpha}, P\right)$. Then for all $\varepsilon>0$, there exists $\delta>0$, such that if $\left(\alpha, \alpha^{\prime}\right)$ is an orbit equivalence with $m\left(\alpha, \alpha^{\prime}\right)<\delta$, then $\left|h\left(T_{\alpha}, P\right)-h\left(T_{\alpha^{\prime}}, P\right)\right|<\varepsilon$. Furthermore, as can be seen by an examination of the proof of this fact, $\delta$ depends only $\varepsilon, m$ and the process $\left(T_{\alpha}, P\right)$ and not on $\alpha$. This fact appears as Lemma 3.13 in [2], and we will refer to it in this way when we use it.

If $\left(\alpha, \alpha^{\prime}\right)$ is an orbit equivalence, then a collapsing of $\alpha^{\prime}$ onto $\alpha$ is an ordering $\bar{\alpha}^{\prime}$ such that $\left(\alpha, \bar{\alpha}^{\prime}\right)$ is a coboundary of a special form. Namely, for almost every $\omega$, the permutation of $\mathbb{Z}$ given by the orbit equivalence on the orbit of $\omega$ is a concatenation of permutations of finite intervals of bounded length. More precisely, there is an increasing sequence of integers $\left\{a_{i}\right\}_{i=-\infty}^{\infty}$ with $\left\{a_{i+1}-a_{i}\right\}_{i=-\infty}^{\infty}$ bounded, such that for each $i$ and each integer $n$,

$$
f_{\omega}^{\alpha, \bar{\alpha}^{\prime}}(n)=\prod_{\omega,\left[a_{i}, a_{i+1}-1\right]}^{\alpha, \bar{\alpha}^{\prime}}(n)-\prod_{\omega,\left[a_{0}, a_{1}-1\right]}^{\alpha, \bar{\alpha}^{\prime}}(0),
$$


where $a_{0} \leq 0 \leq a_{1}-1$. Furthermore, for each $i$,

$$
\prod_{\omega,\left[a_{i}, a_{i+1}-1\right]}^{\alpha, \bar{\alpha}^{\prime}}=\prod_{\omega,\left[a_{i}, a_{i+1}-1\right]}^{\alpha, \alpha^{\prime}} .
$$

Given $\left(\alpha, \alpha^{\prime}\right), \hat{\varepsilon}>0$, and positive integer $K$, there is a collapsing $\bar{\alpha}^{\prime}$ of $\alpha^{\prime}$ onto $\alpha$ such that for a set of $\omega$ of measure greater than $1-\hat{\varepsilon}$ and all $k \in(-K, K), T_{\alpha^{\prime}}^{k}(\omega)=$ $T_{\hat{\alpha}^{\prime}}^{k}(\omega)$. We refer to such a collapsing as an $(\hat{\varepsilon}, K)$-collapsing of $\alpha^{\prime}$ onto $\alpha$. If in addition we are given $\varepsilon>0$, then there is a $\delta$ (depending only on $\varepsilon$ and $m$ ) so that if $m\left(\alpha, \alpha^{\prime}\right)<\delta, \bar{\alpha}^{\prime}$ may be chosen so that $m\left(\alpha, \bar{\alpha}^{\prime}\right)<\varepsilon$. Such a collapsing is called an $(\varepsilon, \hat{\varepsilon}, K)$-collapsing of $\alpha^{\prime}$ onto $\alpha$.

Now suppose that $\left(\alpha, \alpha^{\prime}\right)$ is a coboundary. Then for all finite partitions $P, \hat{\varepsilon}>0$ and positive integers $K$, there is an $(\hat{\varepsilon}, K)$-collapsing $\bar{\alpha}^{\prime}$ of $\alpha^{\prime}$ onto $\alpha$ such that $\left|h\left(T_{\alpha^{\prime}}, P\right)-h\left(T_{\bar{\alpha}^{\prime}}, P\right)\right|<\hat{\varepsilon}$. If in addition we are given $\varepsilon>0$, then there is a $\delta>0$, depending only on $\varepsilon$ and $m$, such that if $m\left(\alpha, \alpha^{\prime}\right)<\delta$, then $\bar{\alpha}^{\prime}$ may be chosen to be an $(\varepsilon, \hat{\varepsilon}, K)$-collapsing satisfying the above entropy condition. This fact appears as Lemma 3.14 in [2], and this is how we will refer to it.

A few additional results will be used repeatedly in our arguments, and we state than here as lemmas for the convenience of the reader.

Lemma A. Let $(T, P)$ be an ergodic process with $h(T, P)>0$. then for all $\varepsilon>0$, there exists a partition $\bar{P}$ sach that $|P-\bar{P}|<2 \varepsilon$ and $h(T, \bar{P})<(1-\varepsilon) h(T, P)$.

Proof: This is Proposition 4.4 of [1].

LEMMA B. (Cf. Lemma 0 in [4].) Let $B$ be an abstract partition. Then for all $\varepsilon>0$ there exists $\xi>0$ such that if $\left(T^{*}, B^{*} \vee H^{*}\right)$ is a process such that $\left|d\left(B^{*}\right)-d(B)\right|<\xi$, $\left|h\left(T^{*},\left.B^{*}\right|_{H^{*}}\right)-H(B)\right|<\xi$, and $h\left(T^{*}\right)>H(\hat{B})+h\left(T^{*}, H^{*}\right)$ then there exists a partition $\hat{B}$ such that $\left|\hat{B}-B^{*}\right|<\varepsilon, d(\hat{B})=d\left(B^{*}\right)$ and $\left(T^{*}, \hat{B}\right)$ is an independent process, independent of $\left(T^{*}, H^{*}\right)$.

Proof. A proof may be found in [3]. See Proposition 2 of that work.

Before proceeding with the proofs of our results, it may be appropriate to comment on the relation between our work here and that of Thouvenot in [4]. Our results generalize those of Thouvenot, and of course we make considerable use of his work, sometimes unavoidably and other times as a matter of convenience. There are, however, some novel aspects of our presentation, even as it pertains to the case where an $m$-equivalence is an isomorphism. In particular, the basic coding argument here (appearing in Lemma 1), when restricted to the isomorphism case, is different from that of Thouvenot (cf. Lemma 4 in [4]). His argument yields stronger conclusions, while our is correspondingly simpler and sufficient to prove the stability theorems.

THEOREM 1. Let $T$ be a transformation m-equivalent to $\hat{T}$, where $\hat{T}$ has the weak Pinsker property. Let $\mathscr{P}$ be a factor of $T$. Then the transformation $(T, \mathscr{P})$ is m-equivalent to a transformation with the weak Pinsker property.

Theorem 1 will follow from repeated application of the following lemma and the results of Thouvenot [4]. 
Lemma 1. Let $T$ and $\hat{T}$ be m-equivalent transformations where $\hat{T}$ has the weak Pinsker property. Let $(\alpha, \hat{\alpha})$ be an m-equivalence such that $T_{\alpha}$ is isomorphic to $T$, and $T_{\bar{\alpha}}$ is isomorphic to $\hat{T}$. Let $\mathscr{P}$ be a factor of $T_{\alpha}, P$ a finite partition in $\mathscr{P}$ and $\varepsilon>0$. Then there is an ordering $\alpha_{1}$, differing from $\alpha$ by a coboundary measurable with respect to $\mathscr{P}$, and finite partitions $B$ and $H_{1}$ in $\mathscr{P}$ such that

(1) $m\left(\alpha, \alpha_{1}\right)<\varepsilon$,

(2) $h\left(T_{\alpha_{1}}, H_{1}\right)<\varepsilon$,

(3) $\left(T_{\alpha_{1}}, B\right)$ is an independent process, independent of $\left(T_{\alpha_{1}}, H_{1}\right)$,

(4) There exists an integer $k$ such that $\left(T_{\alpha_{1}},\left(B \vee H_{1}\right)\right)_{-k}^{k} \supset_{\varepsilon} P$

and

(5) $h\left(T_{\alpha_{1}}, B \vee H_{1}\right)<h\left(T_{\alpha_{1}}\right)$.

Proof. To avoid trivialities, we may assume that $h\left(T_{\alpha}, P\right)>0$. Choose $\delta_{1}<1$ by Lemma 3.14 of [2] with respect to $\varepsilon$ and by Lemma 3.13 of [2] so that $m(\hat{\alpha}, \alpha)<\delta_{1}$ implies $\left|h\left(T_{\hat{\alpha}}, P\right)-h\left(T_{\alpha}, P\right)\right| / h\left(T_{\hat{\alpha}}, P\right)<\varepsilon / 8$. Choose $\delta_{2}$ by axiom (vi) so that if $\alpha_{1}, \alpha_{2}, \alpha_{3}$ are orderings satisfying $m\left(\alpha_{1}, \alpha_{2}\right)<\delta_{1} / 2$ and $m\left(\alpha_{2}, \alpha_{3}\right)<\delta_{2}$ then $m\left(\alpha_{1}, \alpha_{3}\right)<\delta_{1}$. Since $(a, \hat{\alpha})$ is an $m$-equivalence, we may assume that $m(\alpha, \hat{\alpha})<$ $\delta_{1} / 2$.

Since $T_{\hat{\alpha}}$ has the weak Pinsker property, we can apply Lemma A to $\left(T_{\hat{\alpha}}, P\right)$ and apply Proposition 1 of [4] to obtain finite partitions $\hat{B}$ and $\hat{H}$ such that

(6) $\left(T_{\hat{\alpha}}, \hat{B}\right)$ is an independent process, independent of $\left(T_{\hat{\alpha}}, \hat{H}\right)$, and $h\left(T_{\hat{\alpha}}, \hat{H}\right)<\varepsilon$,

(7) $h\left(T_{\hat{\alpha}}, \hat{B} \vee \hat{H}\right)<(1-\varepsilon / 4) h\left(T_{\hat{\alpha}}, P\right)$ and

(8) for some $k,\left(T_{\hat{\alpha}}, \hat{B} \vee \hat{H}\right)_{-k}^{k} \supset_{\varepsilon / 2} P$.

Note that (7) and the choice of $\delta_{1}$ imply that

(9) $h\left(T_{\hat{\alpha}}, \hat{B} \vee \hat{H}\right)<h\left(T_{\alpha}, P\right)-(\varepsilon / 8) h\left(T_{\hat{\alpha}}, P\right)$.

Let $\rho=\varepsilon(2(2 k+1))$. Using Lemma $\mathrm{B}$, choose $\xi>0, \xi<\varepsilon / 100$, so that every process $\left(T^{*}, B^{*} \vee H^{*}\right)$ satisfying

(10) $\left|\operatorname{dist}\left(B^{*}\right)-\operatorname{dist}(\hat{B})\right|<\xi$,

(11) $h\left(T^{*},\left.B^{*}\right|_{H^{*}}\right)-H(\hat{B}) \mid<\xi$ and

(12) $h\left(T^{*}\right)>H(\hat{B})+h\left(T^{*}, H^{*}\right)$

admits a partition $B$ such that

(13) $\left|B-B^{*}\right|<\rho$ and $\left(T^{*}, B \vee H^{*}\right)$ has the same distributions as $\left(T_{\hat{\alpha}}, \hat{B}\right) \times\left(T^{*}, H^{*}\right)$.

Let $\tilde{\alpha}$ be an ordering differing from $\alpha$ by a coboundary and such that $m(\hat{\alpha}, \tilde{\alpha})<\delta_{2}$ and (using Lemma 3.13 of [2]) such that the process $\left(T_{\dot{\alpha}}, \hat{B} \vee \hat{H}\right)$ satisfies

(14) $\left(T_{\tilde{\alpha}}, \hat{B} \vee \hat{H}\right)_{-k}^{k} \supset_{\varepsilon / 2} P$,

(15) $h\left(T_{\tilde{\alpha}}, \hat{H}\right)<\varepsilon$,

(16) $H(\hat{B})+h\left(T_{\dot{\alpha}}, \hat{H}\right)<h\left(T_{\alpha}, P\right)$

(17a) $h\left(T_{\tilde{\alpha}},\left.\hat{B}\right|_{\hat{H}}\right)<H(\hat{B})+\xi$,

(17b) $h\left(T_{\tilde{\alpha}},\left.\hat{B}\right|_{\hat{H}}\right)>H(\hat{B})-\xi / 4$.

Since $m(\tilde{\alpha}, \alpha)<\delta_{1}$ we may invoke Lemma 3.14 of [2] and let $\bar{\alpha}$ be a collapsing of $\tilde{\alpha}$ on $\alpha$ such that conditions (14) through (17b) hold with $T_{\tilde{\alpha}}$ in place of $T_{\tilde{\alpha}}$, and such that $m(\bar{\alpha}, \alpha)<\varepsilon$.

Note that conditions (14) through (17a) will also be satisfied by every process $\left(T^{*}, P^{*} \vee B^{*} \vee H^{*}\right)$ whose distribution of sufficiently great length $n$ is sufficiently close (say to within $\eta)$ of that of $\left(T_{\bar{\alpha}}, P \vee \hat{B} \vee \hat{H}\right)$. In addition, we can choose $n$ 
and $\eta$ so that such processes satisfy

(18) $h\left(T^{*}, H^{*}\right)<h\left(T_{\check{\alpha}}, H\right)+\xi / 4$.

Let $\varphi$ denote the natural isomorphism between $T_{\alpha}$ and $T_{\bar{\alpha}}$ given by the coboundary relating them. Let $\bar{P}, \bar{B}$, and $\bar{H}$ denote the inverse images of $P, \hat{B}$, and $\hat{H}$, respectively, under $\varphi$. Fix $\bar{\delta}>0, \bar{\delta}<\frac{1}{2}$, whose size will be determined in the course of the following argument.

Choose $N$ so large that for all points $\omega$ outside a set of measure less that $\bar{\delta}$ we have (19) $\mu\left(\left(T_{\alpha}, P\right)_{1}^{N}(\omega)\right)=2^{-N\left(h\left(T_{\alpha}, P \pm \bar{\delta}\right)\right.}$,

(20) $\mu\left(\left(T_{\alpha}, \bar{B} \vee \bar{H}\right)(\omega)\right)=2^{-N\left(h\left(T_{\alpha}, \bar{B} \vee \bar{H}\right) \pm \bar{\delta}\right)}$, and

(21) letting $M$ denote the maximum of the lengths of the permutations concatenated to form the collapsing $\bar{\alpha}$, the distribution of $\left(T_{\alpha}, \bar{P} \vee \bar{B} \vee \bar{H}\right)_{1}^{n}$-names along $\left(T_{\alpha}, \bar{P} \vee \bar{B} \vee \bar{H}\right)_{1}^{N}(\omega)$ is within $\eta$ of the actual distribution, even after the initial and final segments of length $2 M$ are altered arbitrarily. It will be convenient to assume that $\eta<\xi$.

Choose $N$ additionally so that $N \bar{\delta}>1$ and $2^{-N \bar{\delta}}<\frac{1}{2}$ and so that $N$ is large enough to satisfy further conditions that will emerge in the following argument.

Let $u=h\left(T_{\alpha}, P\right)-h\left(T_{\alpha}, \bar{B} \vee \bar{H}\right)$, and let $\bar{L}$ be a partition independent of $\left(T_{\alpha}, \bar{P} \vee \bar{B} \vee \bar{H}\right)^{N-1}$ consisting of between $2^{N(u+3 \bar{\delta})}$ and $2^{N(u+4 \bar{\delta})}$ atoms of equal measure. We want to assign to most atoms $p \in\left(T_{\alpha}, P\right)_{0}^{N-1}$ a point $\omega(p) \in p$ such that $\omega(p)$ is in the set $C$ of points satisfying condition (21), and the assignment of names

$$
\left(T_{\alpha}, P\right)_{0}^{N-1}(p) \rightarrow\left(T_{\alpha}, \bar{B} \vee \bar{H} \vee \bar{L}\right)_{0}^{N-1}(\omega(p))
$$

is one-to-one.

Let $\mathfrak{D}$ denote the collection of sets of the form $(\bar{b} \cap \bar{h} \cap \bar{l}) \cap C$ where $\bar{b} \cap \bar{h} \cap \bar{l}$ is an element of $\left(T_{\alpha}, \bar{B} \vee \bar{H} \vee \bar{L}\right)_{0}^{N-1}$ contained in an element $\bar{b} \cap \bar{h}$ of $\left(T_{\alpha}, \bar{B} \vee \bar{H}\right)_{0}^{N-1}$ satisfying condition (20). We have $\mu(\cup \mathfrak{D})>1-\bar{\delta}$. If $d \in \mathfrak{D}$ and $p$ is an element of $\left(T_{\alpha}, P\right)_{0}^{N-1}$ satisfying condition (19), then

$$
\begin{aligned}
\mu(d) / \mu(p) & <\left(2^{-N\left(h\left(T_{\alpha}, B \vee H\right)-\bar{\delta}\right)}\right)\left(2^{-N(u+3 \bar{\delta})}\right)\left(2^{N\left(h\left(T_{\alpha}, P\right)+\bar{\delta}\right.}\right) \\
& =2^{-N(\bar{\delta})}<\frac{1}{2} .
\end{aligned}
$$

It follows that there is a collection $\mathscr{E}$ of atoms $p$ of $\left(T_{\alpha}, P\right)_{0}^{N-1}$ satisfying condition (19), each of which has a fraction at least $1-(\bar{\delta})^{1 / 2}$ of its measure contained in $\bigcup \mathfrak{D}$, and $\mu(\bigcup \mathscr{E})>1-\bar{\delta}-(\bar{\delta})^{1 / 2}$. We may therefore apply the marriage lemma to obtain a one-to-one map $\lambda$ from $\mathscr{E}$ to $\mathscr{D}$ such that for all $p \in \mathscr{E}, \mu(p \cap \lambda(p))>0$. We then define $\omega(p)$ to be an arbitrarily chosen point in $p \cap \lambda(p)$.

We now associate with each $\omega(p)$ (and hence to each $p \in \mathscr{E}$ ) a permutation $\prod_{\omega(p)}$ of $[0, N-1]$, as follows. The interval $[0, N-1]$ is partitioned into disjoint subintervals $I_{j}=\left[i_{j}, i_{j+1}-1\right], j=1,2, \ldots, J_{\omega(p)}$, where the sets $\left\{T_{\alpha}^{i} \omega(p)\right\}_{i \in I_{j}}$ form complete blocks of the orbit change $(\alpha, \bar{\alpha})$, with the possible exception of the first and last of these sets, which may form only a fraction of such a block. For each $j \in\left\{1,2, \ldots, J_{\omega(p)}\right\}$, let $\prod_{j}$ denote $\prod_{\omega(p),\left[i_{j}, i_{j+1}-1\right]}^{(\alpha, \tilde{r})}$. Let $j_{0}=j_{0}(\omega(p))$ be the least integer such that $i_{j_{0}}>M$, and set

$$
\prod_{\omega(p)}=\prod_{0} \circ \prod_{j_{0}} \circ \prod_{j_{0}+1} \circ \cdots \circ \prod_{J_{\omega(p)}-1} \circ \prod_{0}
$$


where $\prod_{0}$ and $\bar{\Pi}_{0}$ denote the identity permutations on the initial and final segments, respectively.

To construct the desired coboundary $\left(\alpha, \alpha_{1}\right)$, let $\bar{N}$ be the integer given by Axiom (v) and used in the construction of $\bar{\alpha}$; namely, a lower bound on the lengths of the permutations concatenated to form $(\alpha, \bar{\alpha})$ in order to insure that $m(\alpha, \bar{\alpha})<\varepsilon$. Let $\tau=\bigcup_{i} T_{\alpha}^{i} A$ be a Rochlin tower in the factor $\left(T_{\alpha}, \mathscr{P}\right)$ of height $N$, base $A$, and measure $1-\bar{\delta}$, and with

$$
\left.\operatorname{dist}\left(T_{\alpha}, P\right)_{0}^{N-1}\right|_{A}=\operatorname{dist}\left(T_{\alpha}, P\right)_{0}^{N-1} \text {. }
$$

Choose $\tau$ also so that for all $\omega \in T_{\alpha}^{N-1}(A)$, the return-time function $r_{\tau}$ satisfies $r_{\tau}(\omega)>\bar{N}$. (Note that this entails an additional condition on $N$.) Now contruct $\alpha_{1}$ by permuting each column in $\tau$ with a name $p \in \mathscr{E}$ by $\prod_{\omega(p)}$ and leaving all other orbit segments untouched. More precisely, if $\omega^{\prime} \in(A \cap p)$ where $p \in \mathscr{E}$, we set, for all $i \in[0, N-1], f_{\omega^{\prime}}^{\left(\alpha, \alpha_{i}\right)}(i)=\prod_{\omega(p)}(i)$. If $\omega \in A$, and $\omega \notin \bigcup \mathscr{E}$ and $i \in[0, N-1]$, we set $f_{\omega^{\prime}}^{\left(\alpha, \alpha_{1}\right)}(i)=i$, and similarly, if $\omega \in T_{\alpha}^{N-1}(A)$ and $i \in\left[1, r_{\tau}(\omega)-1\right]$, we set $f_{\omega^{\prime}}^{\left(\alpha, \alpha_{1}\right)}(i)=i$.

It is now clear that $m\left(\alpha, \alpha_{1}\right)<\varepsilon$. Indeed, for almost every $\omega$, the forward and backward orbits of $\omega$ infinitely often contain a block of length $M$, and between these occurrences we see concatenated permutations of size less than $\delta_{1}$ on intervals of length between $\bar{N}$ and $M$, together with possibly longer intervals on which the identity permutation acts. But these longer blocks, exceeding $\bar{N}$ in length, can be regarded as sequences of blocks of lengths between $\bar{N}$ and $2 \bar{N}(<M)$ on all of which the identity acts, so that, as in the construction of $\bar{\alpha}, m\left(\alpha, \alpha_{1}\right)<\varepsilon$.

We define partitions $\overline{\bar{B}}, \overline{\bar{H}}, \overline{\bar{L}}$ and $\overline{\bar{P}}$, measurable with respect to the factor $\left(T_{\alpha}, \mathscr{P}\right)$ as follows. For $\omega^{\prime} \in A \cap p$, where $p \in \mathscr{E}$, and for $i \in[0, N-1]$, we set $\overline{\bar{B}}\left(T_{\alpha}^{i}\left(\omega^{\prime}\right)\right)=\bar{B}\left(T_{\alpha}^{i}(\omega(p))\right)$, and we put the rest of the space in a single atom of $\overline{\bar{B}}$. The partitions $\bar{H}$ and $\overline{\bar{P}}$ are defined similarly. The partition $\bar{L}$ is defined by setting $\bar{L}\left(\omega^{\prime}\right)=\bar{L}(\omega(p))$, for $\omega \in A \cap p, p \in \mathscr{E}$, and all the rest of the space in a single, additional atom of $\bar{L}$. Let $\varphi_{1}$ be the natural isomorphism between $T_{\alpha}$ and $T_{\alpha_{1}}$, and let $B_{1}=\varphi_{1}(\overline{\bar{B}}), H_{1}=\varphi_{1}(\overline{\bar{H}}), L_{1}=\varphi_{1}(\overline{\bar{L}})$, and $P_{1}=\varphi_{1}(\overline{\bar{P}})$. Let $P_{2}=\varphi_{1}(P)$. Now for every $\omega^{\prime} \in A \cap p$, where $p \in \mathscr{E}$,

$$
\begin{aligned}
\left(T_{\alpha_{1}}, P_{1} \vee B_{1} \vee H_{1}\right)_{0}^{N-1}\left(\omega^{\prime}\right) & =\left(T_{\alpha}, \overline{\bar{P}} \vee \overline{\bar{B}} \vee \overline{\bar{H}}\right)_{0}^{N-1}\left(\omega^{\prime}\right) \\
& =\left(T_{\alpha}, \bar{P} \vee \bar{B} \vee \bar{H}\right)_{0}^{N-1}(\omega(p))
\end{aligned}
$$

and

agrees with

$$
\left(T_{\alpha_{1}}, P \vee B_{1} \vee H_{1}\right)_{0}^{N-1}\left(\omega^{\prime}\right)
$$

$$
\left(T_{\alpha_{1}}, P_{1} \vee B_{1} \vee H_{1}\right)_{0}^{N-1}\left(\omega^{\prime}\right)
$$

except perhaps in the initial or final segments of lengths $2 M$. Since these names satisfy condition 21 , and the processes $\left(T_{\alpha}, \bar{P} \vee \bar{B} \vee \bar{H}\right)$ and $\left(T_{\bar{\alpha}}, P \vee \hat{B} \vee \hat{H}\right)$ have the same distributions, we get that if $\bar{\delta}$ is sufficiently small,

$$
\left|\operatorname{dist}\left(T_{\alpha_{1}}, P \vee B_{1} \vee H_{1}\right)_{0}^{n-1}-\operatorname{dist}\left(T_{\bar{\alpha}}, P \vee \hat{B} \vee \hat{H}\right)_{0}^{n-1}\right|<\eta
$$

so that the conditions corresponding to (14), (15), (16), (17a) and (18) hold. It remains to verify $(17 \mathrm{~b})$. 
If $\bar{\delta}$ was chosen sufficiently small, then $P_{2}$ is so well approximated by sets in $\left(T_{\alpha_{1}}, B_{1} \vee H_{1} \vee L_{1}\right)_{-N}^{N}$ that

$$
h\left(T_{\alpha_{1}}, B_{1} \vee H_{1} \vee L_{1}\right)>h\left(T_{\alpha_{1}}, P_{2}\right)-\xi / 4=h\left(T_{\alpha}, P\right)-\xi / 4 .
$$

However, if $N$ was chosen sufficiently large, then $H\left(L_{1}\right)<u+4 \bar{\delta}$, so that

$$
\begin{aligned}
h\left(T_{\alpha_{1}}, B_{1} \vee H_{1}\right)>h\left(T_{\alpha}, P\right)-u-\xi / 4-4 \bar{\delta} & =h\left(T_{\alpha}, \bar{B} \vee \bar{H}\right)-\xi / 4-4 \bar{\delta} \\
& =h\left(T_{\bar{\alpha}}, \hat{B} \vee \hat{H}\right)-\xi / 4-4 \bar{\delta} .
\end{aligned}
$$

On the other hand we have by condition (18) that $h\left(T_{\alpha_{1}}, H_{1}\right)<h\left(T_{\bar{\alpha}}, \hat{H}\right)+\xi / 4$, so that

$$
h\left(T_{\alpha_{1}}, B_{1} \vee H_{1}\right)-h\left(T_{\alpha_{1}}, H_{1}\right)>h\left(T_{\bar{\alpha}}, \hat{B} \vee \hat{H}\right)-h\left(T_{\bar{\alpha}}, \hat{H}\right)-\xi / 2-4 \bar{\delta},
$$

which, if $\bar{\delta}$ is sufficiently small, establishes (17b). Note that we also have that, (providing $\bar{\delta}$ is sufficiently small), $\left|\operatorname{dist}\left(B_{1}\right)-\operatorname{dist}(\hat{B})\right|<\xi$. It follows that there is a partition $B$ such that $\left(T_{\alpha_{1}}, B\right)$ is an independent process, independent of $\left(T_{\alpha_{1}}, H_{1}\right)$, with dist $(B)=\operatorname{dist}(\hat{B})$ and $\left|B-B_{1}\right|<\rho$. We therefore have $\left(T_{\alpha_{1}}, B \vee H_{1}\right)_{-k}^{k} \supset_{\varepsilon} P$. Since condition (16) gives

$$
h\left(T_{\alpha_{1}}, B \vee H_{1}\right)=H(\hat{B})+h\left(T_{\alpha_{1}}, H_{1}\right)<h\left(T_{\alpha}, P\right)=h\left(T_{\alpha_{1}}, P_{2}\right)<h\left(T_{\alpha_{1}}\right),
$$

we are done.

Proof of Theorem 1. Let $(\alpha, \hat{\alpha})$ be an $m$-equivalence such that $T_{\alpha}$ is isomorphic to $T$ and $T_{\hat{\alpha}}$ is isomorphic to $\hat{T}$. Let $\left\{P_{i}\right\}_{i=1}^{\infty}$ be an increasing sequence of finite partitions such that $\bigvee_{i=1}^{\infty} P_{i}=\mathscr{P}$. We will construct an $m$-equivalence $\left(\alpha, \alpha^{\prime}\right)$, measurable with respect to $\mathscr{P}$, such that for each $i$, there exist finite partitions $\bar{B}_{i}$ and $H_{i}$ such that (22) $\left(\bar{B}_{i} \vee H_{i}\right)_{T_{\alpha^{\prime}}} \supset_{\varepsilon_{i}} P_{i}$,

(23) $h\left(T_{\alpha^{\prime}}, H_{i}\right)<\varepsilon_{i}$,

(24) the process $\left(T_{\alpha^{\prime}}, \bar{B}_{i}\right)$ is independent and independent of $\left(T_{\alpha^{\prime}}, H_{i}\right)$, where $\lim _{i \rightarrow \infty} \varepsilon_{i}=0$. Since the $P_{i}$ increase and the $\varepsilon_{i} \rightarrow 0$, it follows from the work of Thouvenot [4] that each $\left(T_{\alpha^{\prime}}, P_{i}\right)$ has the weak Pinsker property, and therefore, since $\bigvee_{i=1}^{\infty} P_{i}=\mathscr{P}$, so does the transformation $\left(T_{\alpha}, \mathscr{P}\right)$ itself.

Fix a sequence $\left\{\varepsilon_{i}\right\}_{i=1}^{\infty}$ with $\lim _{i \rightarrow \infty} \varepsilon_{i}=0$. Apply Lemma 1 to get an ordering $\alpha_{1}$, differing from $\alpha$ by a coboundary measurable with respect to $\mathscr{P}$, such that there exist finite partitions $B_{1}$ and $H_{1}$ and an integer $n_{1}$ with

$$
\left(T_{\alpha_{1}}, B_{1} \vee H_{1}\right)_{-n_{1}}^{n_{1}} \supset \varepsilon_{\varepsilon_{1} / 2} P_{1}, h\left(T_{\alpha_{1}}, H_{1}\right)<\varepsilon_{1},
$$

the process $\left(T_{\alpha_{1}}, B_{1}\right)$ independent and independent of $\left(T_{\alpha_{1}}, H_{1}\right)$ and $h\left(T_{\alpha_{1}}, B_{1} \vee H_{1}\right)<h\left(T_{\alpha_{1}}\right)$. We now apply Lemma 1 iteratively as follows. After the $(i-1)$ st application we have a sequence of orderings $\left\{\alpha_{j}\right\}_{j=1}^{i-1}$, each $\left(\alpha_{j-1}, \alpha_{j}\right)$ a coboundary, and for all $j \in\{1,2, \ldots, i-1\}$, partitions $B_{j}$ and $H_{j}$ and integers $n_{j}$ and constants $\xi_{j}$ and $\delta_{j}$ such that

(25) $\left(T_{\alpha_{j}}, B_{j} \vee H_{j}\right)_{-n_{n_{j}}}^{n_{j}} \supset_{\varepsilon_{j} / 2} P_{j}$,

(26) $h\left(T_{\alpha_{i}}, H_{j}\right)<\varepsilon_{j}$,

(27) $\left(T_{\alpha_{i}}, B_{j}\right)$ is an independent process, independent of $\left(T_{\alpha_{j}}, H_{j}\right)$ and

(28) $h\left(T_{\alpha_{i}}, B_{j} \vee H_{j}\right)<h\left(T_{\alpha_{i}}\right)$.

Let $\rho_{j}=\varepsilon_{j} / 2\left(2 n_{j}+1\right)$. Using Lemma (B), the constant $\xi_{j}$ is chosen (independent of 
i) so that every process $\left(T^{\prime}, B^{\prime} \vee H^{\prime}\right)$ such that

(29) $\left|\operatorname{dist}\left(B^{\prime}\right)-\operatorname{dist}\left(B_{j}\right)\right|<\xi_{j}$,

(30) $\left|h\left(T^{\prime},\left.B^{\prime}\right|_{H^{\prime}}\right)-H\left(B_{j}\right)\right|<\xi_{j}$ and

(31) $H\left(B_{j}\right)+h\left(T^{\prime}, H^{\prime}\right)<h\left(T^{\prime}\right)$

admits a partition $\bar{B}_{j}$ such that $\left|\bar{B}_{j}-B_{j}\right|<\rho_{j}$ and $\left(T^{\prime}, \bar{B}_{j} \vee H^{\prime}\right)$ has the same distributions as $\left(T_{\alpha_{j}}, B_{j} \vee H_{j}\right)$. The constant $\delta_{j}$ is chosen (independent of $i$ ) so that $\delta_{j}<1 / j$ and if $m\left(\alpha^{\prime}, \alpha_{j}\right)<\delta_{j}$, then

(32) $\left(T_{\alpha^{\prime}}, B_{j} \vee H_{j}\right)_{-n_{j}}^{n_{j}} \supset_{\varepsilon_{j} / 2} P_{j}$,

(33) $h\left(T_{\alpha^{\prime}}, H_{j}\right)<\varepsilon_{j}$,

(34) $\left|h\left(T_{\alpha^{\prime}},\left.B_{j}\right|_{H_{j}}\right)-H\left(B_{j}\right)\right|<\xi_{j}$ and

(35) $H\left(B_{j}\right)+h\left(T_{\alpha^{\prime}}, H_{j}\right)<h\left(T_{\alpha^{\prime}}\right)$.

In addition, we have, for each $j<i, m\left(\alpha_{j}, \alpha_{i-1}\right)<\left(1-2^{-(i-j-1)}\right) \delta_{j}$. Finally, for each $j \in\{2,3, \ldots, i-1\}$, we have an integer $K_{j}$ (whose significance will become clear in what follows).

We now choose an integer $K_{i}>K_{i-1}$ and, for all $j<i-1$ and almost every $\omega$, integers $a_{j, i}(\omega)<-i<i<b_{j, i}(\omega)$ so that

$$
m\left(\prod_{\omega}^{\left(\alpha_{j} \alpha_{i-1}\right)}\left[a_{j, i}(\omega), b_{j, i}(\omega)\right]\right)<\delta_{j},
$$

and for all $\omega$ outside a set $A_{i, 1}$ of measure less than $2^{-i}$,

(36) $f_{\omega}^{\alpha, \alpha_{i-1}}[-i, i] \subset\left[-K_{i}, K_{i}\right]$ and

(37) $f_{\omega^{\prime}}^{\alpha_{j}, \alpha_{i-1}}\left[a_{j, i}(\omega), b_{j, i}(\omega)\right] \subset\left[-K_{i}, K_{i}\right]$.

Choose $\eta_{i}$ so that if $m\left(\alpha_{i-1}, \alpha_{i}\right)<\eta_{i}$ then for all $\omega$ outside a set $A_{i, 2}$ of measure less than $2^{-i}, f_{\omega}^{\alpha_{i-1} \cdot \alpha_{i}}(n)=n$, for all $n \in\left[-K_{i}, K_{i}\right]$, and so that for all

$$
j<i-1, m\left(\alpha_{j}, \alpha_{i}\right)<\left(1-2^{-(i-j)}\right) \delta_{j} .
$$

Since $T_{\alpha_{i-1}}$ is $m$-equivalent to $T_{\hat{\alpha}}$, we may apply Lemma 1 to get $\alpha_{i}$, differing from $\alpha_{i-1}$ by a coboundary measurable with respect to $\mathscr{P}$, so that $m\left(\alpha_{i-1}, \alpha_{i}\right)<\eta_{i}$, and so that there exist finite partitions $B_{i}$ and $H_{i}$ and $n_{i}$ such that

$$
\left(T_{\alpha_{i}}, B_{i} \vee H_{i}\right)_{-n_{i}}^{n_{i}} \supset_{\varepsilon_{i} / 2} P_{i}, h\left(T_{\alpha_{i}}, H_{i}\right)<\varepsilon_{i},\left(T_{\alpha_{i}}, B_{i}\right)
$$

is independent and independent of $\left(T_{\alpha_{i}}, H_{i}\right)$, and $h\left(T_{\alpha_{i}}, B_{i} \vee H_{i}\right)<h\left(T_{\alpha_{i}}\right)$.

We now verify that the orderings $\alpha_{i}$ converge to the desired ordering $\alpha^{\prime}$. First we check that the $\alpha_{i}$ converge to an ordering with the same orbits as $\alpha$. Setting $A_{i}=A_{i, 1} \cup A_{i, 2}$, we have $\mu\left(\bigcup_{k=1}^{\infty}\left(\bigcap_{i=k}^{\infty} A_{i}^{c}\right)\right)=1$ so for almost every $\omega$ and for all $n$, there exists a $k>|n|$ such that $\omega \in \bigcap_{i=k}^{\infty} A_{i}^{c}$. Consequently, for all $m \geq k$,

$$
\alpha_{k-1}\left(\omega, T_{\alpha}^{n}(\omega)\right)=\alpha_{m}\left(\omega, T_{\alpha}^{n}(\omega)\right),
$$

so that $\lim _{m \rightarrow \infty} \alpha_{m}\left(\omega, T_{\alpha}^{n}(\omega)\right)$ exists. On the other hand, for almost every $\omega$ and for all $n$, there exists a $k$ such that $K_{k}>|n|$ and $\omega \in \bigcap_{i=k}^{\infty} A_{i}^{c}$. Therefore, $n=$ $\lim _{m \rightarrow \infty} \alpha_{m}\left(\omega, T_{\alpha_{k-1}}(\omega)\right)$, so $\alpha^{\prime}$ gives an ordering with complete orbits. It is clear from the construction that the orbit equivalence $\left(\alpha, \alpha^{\prime}\right)$ is measurable with respect to $\mathscr{P}$.

Next we check that $\left(\alpha, \alpha^{\prime}\right)$ is an $m$-equivalence. Since each $\left(\alpha, \alpha_{j}\right)$ is a coboundary and the $\delta_{i} \rightarrow 0$, it is sufficient to check that for all $j, m\left(\alpha_{j}, \alpha^{\prime}\right)<\delta_{j}$. But for all $j$, and 
almost every $\omega$ and all $n>0$, there exists a $k>n$ such that $\omega \in \bigcap_{i=k}^{\infty} A_{i}^{c}$. Therefore,

$$
m\left(\prod_{\omega}^{\alpha_{j}, \alpha^{\prime}}\left[a_{k, j}(\omega), b_{k, j}(\omega)\right]\right)=m\left(\prod_{\omega}^{\alpha_{j} \alpha_{k-1}}\left[a_{k, j}(\omega), b_{k, j}(\omega)\right]\right)<\delta_{j} .
$$

Since $\min \left\{-a_{k, j}(\omega), b_{k, j}(\omega)\right\}>k>n$, we get $m\left(\alpha_{j}, \alpha^{\prime}\right)<\delta_{j}$.

Now by the choice of $\delta_{j}$, conditions (32), (33), (34) and (35) are satisfied. Therefore, by the choice of $\xi_{j}$ we get, for each $j$, a partition $\bar{B}_{j}$ satisfying $\left|\bar{B}_{j}-B_{j}\right|<\rho_{j}$ and such that $\left(T_{\alpha^{\prime}}, \bar{B}_{j} \vee H_{j}\right)$ has the same distributions as $\left(T_{\alpha_{j}}, B_{j} \vee H_{j}\right)$. By the choice of $\rho_{j}$, this gives $\left(T_{\alpha^{\prime}}, \bar{B}_{j} \vee H_{j}\right)_{-n_{j}}^{n_{j}} \supset_{\varepsilon_{j}} P_{j}$ and we are done.

We now show that the preceding arguments can be adapted to yield the $\bar{m}$-stability of $m$-equivalence to the weak Pinsker property.

Definition. Given processes $(S, Q)$ and $(\hat{S}, \hat{Q})$, we say $\bar{m}((S, Q),(\hat{S}, \hat{Q}))<\varepsilon$ if there exists an $m$-equivalence $(\alpha, \hat{\alpha})$ with partitions $P$ and $\hat{P}$ such that $\left(T_{\alpha}, P\right)=(S, Q)$, $\left(T_{\hat{\alpha}}, \hat{P}\right)=(\hat{S}, \hat{Q}),|P-\hat{P}|<\varepsilon$, and $m(\alpha, \hat{\alpha})<\varepsilon$. (The equality of processes here means equality of their distributions).

We refer to the data $(\alpha, \hat{\alpha}, P, \hat{P})$ as an $\bar{m}$-joining (or an $\varepsilon-\tilde{m}$-joining) of the processes $(S, Q)$ and $(\hat{S}, \hat{Q})$. Note that this notion of an $\tilde{m}$-joining is less restrictive than that used in the presentation of the $m$-equivalence theorem in [2].

THEOREM 2. Let $(S, Q)$ be a process such that for all $\varepsilon>0$, there exists a process $(\hat{S}, \hat{Q})$ such that $(\hat{S}, \hat{Q})$ has the weak Pinsker property and $\bar{m}((S, Q),(\hat{S}, \hat{Q}))<\varepsilon$. Then $(S, Q)$ is m-equivalent to a transformation with the weak Pinsker property.

Remark. Alternatively, we might require only that each $(\hat{S}, \hat{Q})$ is $m$-equivalent to a transformation with the weak Pinsker property, but it is easy to see that such hypotheses imply the hypotheses as stated.

Proof. We first note that for every finite partition $R$ in $(S, Q)$ and every $\varepsilon>0$ there is a process $(\hat{S}, \hat{R})$ such that $(\hat{S}, \hat{R})$ has the weak Pinsker property and $\bar{m}((S, R)$, $(\hat{S}, \hat{R}))<\varepsilon$. This follows easily from the hypotheses and Axiom (iv).

Now fix a partition $R$ such that $h(S, R)>0$ and fix $\varepsilon>0$. Choose $\delta_{1}<1$ by Lemma 3.13 of [2] so that if $m(\hat{\alpha}, \alpha)<\delta_{1},\left(T_{\alpha}, P\right)=(S, R)$ and $|P-\hat{P}|<\delta_{1}$ then $\left|h\left(T_{\hat{\alpha}}, \hat{P}\right)-h\left(T_{\alpha}, P\right)\right| / h\left(T_{\hat{\alpha}}, \hat{P}\right)<\varepsilon / 8$. Choose $\delta_{1}$ additionally by Lemma 3.14 of [2] with respect to $\varepsilon$. Here (and subsequently) we use the fact that this choice of $\delta_{1}$ with respect to Lemma 3.13 depends only on the process $(S, R)$ and on $m$ and on the degree of approximation desired, and not on the particular ordering $\alpha$. Now let $(\alpha, \hat{\alpha}, P, \hat{P})$ be a $\delta_{1} / 2-\bar{m}$-joining of $(S, R)$ and a weak-Pinsker process $(\hat{S}, \hat{R})$. We then have

$$
h\left(T_{\hat{\alpha}}, \hat{P}\right)<h\left(T_{\alpha}, P\right)+(\varepsilon / 8) h\left(T_{\hat{\alpha}}, \hat{P}\right)
$$

so using Lemma $A$ and the results of [4] we can choose partitions $\hat{B}$ and $\hat{H}$ in $(\hat{P})_{T_{\tilde{\alpha}}}$ such that $h\left(T_{\hat{\alpha}}, \hat{H}\right)<\varepsilon,\left(T_{\hat{\alpha}}, \hat{B}\right)$ is independent and independent of $\left(T_{\hat{\alpha}}, \hat{H}\right)$, for some $k,\left(T_{\hat{\alpha}}, \hat{B} \vee \hat{H}\right)_{-k}^{k} \supset_{\varepsilon / 2} P$, and $h\left(T_{\hat{\alpha}}, \hat{B} \vee \hat{H}\right)<h\left(T_{\alpha}, P\right)-(\varepsilon / 8) h\left(T_{\hat{\alpha}}, \hat{P}\right)$.

We can now argue exactly as in the proof of Lemma 1 that there is an ordering $\alpha_{1}$, differing from $\alpha$ by a coboundary measurable with respect to $\left(T_{\alpha}, P\right)$, with $m\left(\alpha, \alpha_{1}\right)<\varepsilon$ and partitions $B_{1}$ and $H_{1}$ satisfying conditions (2) through (5) of the 
conclusions of Lemma 1 . We may of course view the coboundary change $\left(\alpha, \alpha_{1}\right)$ as a coboundary change of $S$, measurable with respect to $(S, Q)$.

Since $\varepsilon$ and $R$ were arbitrarily chosen, and every transformation differing from $S$ by a coboundary is isomorphic to $S$, we can fix an increasing sequence of partitions $\left\{Q_{i}\right\}_{i=1}^{\infty}$ such that $\bigvee_{i=1}^{\infty} Q_{i}=(Q)_{S}$ and a sequence $\left\{\varepsilon_{i}\right\}_{i=1}^{\infty}$ with $\lim _{i \rightarrow \infty} \varepsilon_{i}=0$, and argue exactly as in the proof of Theorem 1 to construct a sequence of coboundary changes of $S$, all measurable with respect to $(S, Q)$, and converging to an $m$-equivalence between $(S, Q)$ and a transformation with the weak-Pinsker property.

With a little more care in the previous arguments, it is possible to obtain a more general result which contains the previous two. We could have begun with this result and deduced the first two theorems as corollaries, but we felt the arguments would have been less clear with such a presentation.

THEOREM 3. Let $(S, Q)$ be a process such that for all $\varepsilon>0$, there exists a process $(\hat{S}, \hat{Q})$ such that $\bar{m}((S, Q),(\hat{S}, \hat{Q}))<\varepsilon$, and such that there is a process $\left(S_{0}, B_{0} \vee H_{0} \vee\right.$ $\left.P_{0}\right)$ where $h\left(S_{0}, H_{0}\right)<\varepsilon,\left(S_{0}, B_{0}\right)$ is independent and independent of $\left(S_{0}, H_{0}\right)$, $\left(S_{0}, P_{0}\right)=(\hat{S}, \hat{Q})$, and $P_{0} \subset\left(B_{0} \vee H_{0}\right)_{S_{0}}$. Then $(S, Q)$ is m-equivalent to a transformation with the weak-Pinsker property.

Proof. As in the proof of Theorem 2, we note that for all partitions $R$ in $(S, Q)$ and for all $\varepsilon>0$, there is a process $(\hat{S}, \hat{R})$ bearing the same relation to $(S, R)$ as the process $(\hat{S}, \hat{Q})$ of the hypotheses does to $(S, Q)$.

Also as we have seen, it is sufficient to show that for every $R$ in $(S, Q)$ and every $\varepsilon>0$, there is a transformation $S_{1}$, differing from $S$ by a coboundary of $m$-size less than $\varepsilon$ and admitting partitions $B_{1}$ and $H_{1}$ such that $h\left(S_{1}, H_{1}\right)<\varepsilon,\left(S_{1}, B_{1}\right)$ is independent and independent of $\left(S_{1}, H_{1}\right),\left(S_{1}, B_{1} \vee H_{1}\right) \supset_{\varepsilon} R$, and $h\left(S_{1}, B_{1} \vee H_{1}\right)<$ $h\left(S_{1}\right)$. Fix such an $R$ and $\varepsilon$, (and assume that $0<h(S, R)$ ). Using Lemma 3.13 of [2], choose $\delta_{1}$ so that if

then

$$
m(\hat{\alpha}, \alpha)<\delta_{1},\left(T_{\alpha}, P\right)=(S, R) \quad \text { and } \quad|P-\hat{P}|<\delta_{1}
$$

$$
\left|h\left(T_{\hat{\alpha}}, \hat{P}\right)-h\left(T_{\alpha}, P\right)\right| / h\left(T_{\hat{\alpha}}, \hat{P}\right)<\varepsilon / 8
$$

and by Lemma 3.14 of [2] with respect to $\varepsilon$. Let $(\hat{S}, \hat{R})$ be a process such that $\bar{m}((S, R),(\hat{S}, \hat{R}))<\delta_{1} / 2$, and the $\bar{m}$-distance is realized by a $\delta_{1} / 2-\bar{m}$-joining $(\alpha, \hat{\alpha}, P, \hat{P})$ such that $(\hat{S}, \hat{R})$ is a factor of a process $\left(S_{0}, B_{0} \vee H_{0} \vee R_{0}\right)$ as in the hypotheses with

$$
h\left(S_{0}, H_{0}\right)<\min \left\{(\varepsilon / 16) h\left(S_{0}, R_{0}\right), \varepsilon\right\} .
$$

Let $R_{1}$ be a partition in $\left(R_{0}\right)_{S_{0}}$ such that $\left|R_{1}-R_{0}\right|<\varepsilon / 2$ and $h\left(S_{0}, R_{1}\right)<$ $(1-\varepsilon / 4) h\left(S_{0}, R_{0}\right)$. Then $h\left(S_{0}, R_{1} \vee H_{0}\right)<h\left(T_{\alpha}, P\right)-(\varepsilon / 16) h\left(S_{0}, R_{0}\right)$. Since $R_{1}$ is $H_{0}$-relatively finitely determined, there is a partition $B_{1}$ in $\left(R_{1} \vee H_{0}\right)_{S_{0}}$ such that $\left(S_{0}, B_{1}\right)$ is independent and independent of $\left(S_{0}, H_{0}\right)$ and $\left(B_{1} \vee H_{0}\right)_{S_{0}}=\left(R_{1} \vee H_{0}\right)_{S_{0}}$. We can now apply the coding technique used in the proof of Lemma 1 to the process $\left(S_{0}, B_{1} \vee H_{0} \vee R_{0}\right)$ (in the case where an $m$-equivalence is an isomorphism) to obtain partitions $B_{1}^{\prime}$ and $H_{0}^{\prime}$ in $\left(R_{0}\right)_{S_{0}}$ such that $h\left(S_{0}, H_{0}^{\prime}\right)<\varepsilon,\left(S_{0}, B_{1}^{\prime}\right)$ is independent and independent of $\left(S_{0}, H_{0}^{\prime}\right), \quad\left(B_{1}^{\prime} \vee H_{0}^{\prime}\right)_{S_{0}} \supset_{\varepsilon / 2} R_{0}, \quad$ and $\quad h\left(S_{0}, B_{1}^{\prime} \vee H_{0}^{\prime}\right)<$ 
$h\left(S_{0}, R_{0}\right)-(\varepsilon / 16) h\left(S_{0}, R_{0}\right)$. Now let $\hat{B}$ and $\hat{H}$ be partitions in $(\hat{P})_{T_{\vec{\alpha}}}$ such that $\left(T_{\hat{\alpha}}, \hat{B} \vee \hat{H} \vee \hat{P}\right)=\left(S_{0}, B_{1}^{\prime} \vee H_{0}^{\prime} \vee R_{0}\right)$. We can therefore apply the arguments of Lemma 1 again, this time to the $\bar{m}$-joining $(a, P, \hat{\alpha}, \hat{P})$ and the processes $\left(T_{\alpha}, P\right)$ and $\left(T_{\hat{\alpha}}, \hat{B} \vee \hat{H} \vee \hat{P}\right)$ to get an ordering $\alpha_{1}$, differing from $\alpha$ by a coboundary measurable with respect to $\left(T_{\alpha}, P\right)$, and partitions $B$ and $H$ in $(P)_{T_{\alpha_{1}}}$ such that $m\left(\alpha, \alpha_{1}\right)<\varepsilon, h\left(T_{\alpha_{1}}, H\right)<\varepsilon,\left(T_{\alpha_{1}}, B\right)$ is independent and independent of $\left(T_{\alpha_{1}}, H\right)$, $(B \vee H)_{T_{\alpha_{1}}} \supset_{\varepsilon} P$, and $h\left(T_{\alpha_{1}}, B \vee H\right)<h\left(T_{\alpha_{1}}\right)$. Identifying $\left(T_{\alpha}, P\right)$ with $(S, R)$, we obtain the desired transformation $S_{1}$ and partitions $B_{1}$ and $H_{1}$ and the proof is complete.

\section{REFEREN C ES}

[1] D. S. Ornstein, D. J. Rudolph \& Benjamin Weiss. Equivalence of measure preserving transformations. Mem. Amer. Math. Soc. 37(262) (1982).

[2] D. J. Rudolph. Restricted orbit equivalence. Mem. Amer. Math. Soc. 54(323) (1985).

[3] J.-P. Thouvenot. Quelques propriétés des systèmes dynamiques qui se décomposent en un produit de deux systèmes dont l'un est un schema de Bernoulli. Isr. J. Math. 21 (1975), 177-207.

[4] J.-P. Thouvenot. On the stability of the weak Pinsker property. Isr. J. Math. 27 (1977), 150-162. 